\title{
Laryngeal Tuberculosis: A Diagnosis that Should Be Remembered
}

\author{
Kiyoko Kitahara, Yukihiro Yano, Masahide Mori and Soichiro Yokota
}

Key words: aryepiglottic fold, epiglottis, laryngeal tuberculosis

(Intern Med 51: 2841-2842, 2012)

(DOI: 10.2169/internalmedicine.51.8503)
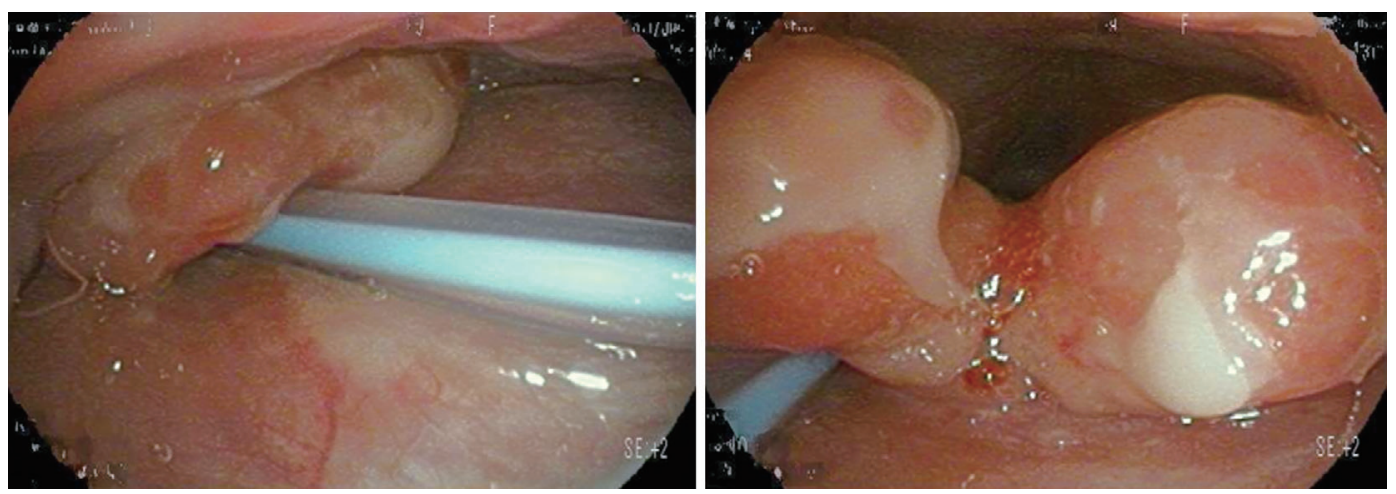

Picture 1.
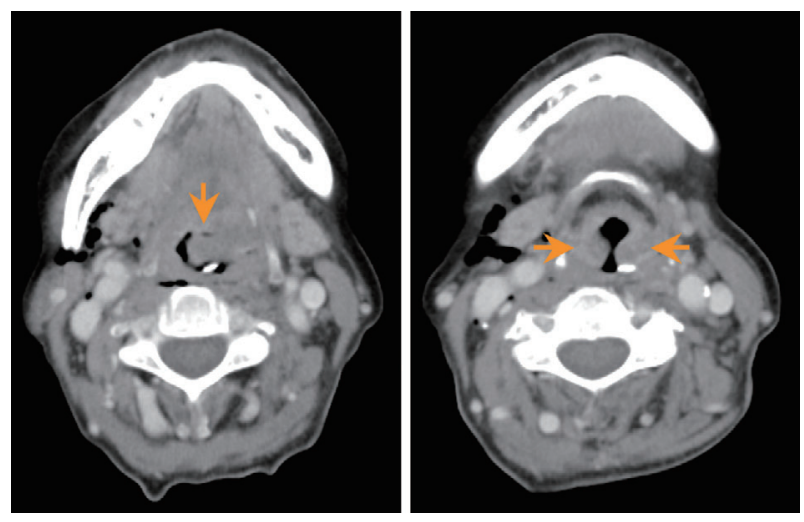

Picture 2.

A 70-year-old woman presented at a local clinic with pharyngodynia, dysphagia and hoarseness with a duration of over three months. Chest X-rays revealed abnormalities and the patient's sputum was positive for tuberculosis. Pulmonary tuberculosis was diagnosed and she was thereafter referred to our hospital. Bronchofiberscopy revealed the presence of abnormalities in the larynx. The epiglottis and arye- piglottic folds were found to be swollen and coated with fur (Picture 1). A CT scan confirmed these findings (Picture 2). Histopathology of the epiglottis revealed the presence of a necrotizing granuloma positive for Ziehl-Neelsen staining, and thus confirming a diagnosis of laryngeal tuberculosis.

In the past, laryngeal tuberculosis was a major complication of advanced pulmonary tuberculosis; however, the incidence of this condition has greatly decreased with the advent of anti-tuberculosis drugs. Unfortunately, the incidence of laryngeal tuberculosis is rising again in accordance with an increasing incidence of tuberculosis due to immunosuppressive diseases and treatments $(1,2)$. Physicians should therefore include laryngeal tuberculosis in the differential diagnosis of patients presenting with laryngeal disorders.

The authors state that they have no Conflict of Interest (COI).

\section{References}

1. Nishiike S, Irifune M, Doi K, Sawada T, Kubo T. Laryngeal tuberculosis: a report of 15 cases. Ann Otol Rhinol Laryngol 111: 916-918, 2002. 
Intern Med 51: 2841-2842, 2012 DOI: 10.2169/internalmedicine.51.8503

2. Lim JY, Kim KM, Choi EC, Kim YH, Kim HS, Choi HS. Current

Eur Arch Otorhinolaryngol 263: 838-842, 2006. clinical propensity of laryngeal tuberculosis: review of 60 cases.

(C) 2012 The Japanese Society of Internal Medicine http://www.naika.or.jp/imonline/index.html 